\title{
Correction: Glucose disturbances, cognitive deficits and white matter abnormalities in first-episode drug-naive schizophrenia
}

\author{
Xiangyang Zhang ${ }^{1,2,3} \cdot$ Mi Yang $^{4,5} \cdot$ Xiangdong Du $^{6} \cdot$ Wei Liao $^{5} \cdot$ Dachun Chen $^{7} \cdot$ Fengmei Fan $^{7} \cdot$ Meihong Xiu $^{7}$.

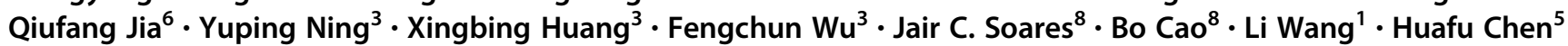

Published online: 22 October 2019

๑) Springer Nature Limited 2019

\section{Correction to: Molecular Psychiatry}

https://doi.org/10.1038/s41380-019-0478-1

published online 13 August 2019

In the original version of this article, some of the values in Table 2 were incorrect. The number of male/female patients was inadvertently misstated as ' $61 / 59$ ', rather than ' $16 / 23$ '.
The 'F/ $\mathrm{X}^{2}$, value, and ' $p$-value' for 'Males/females' were also incorrectly stated as ' 0.32 ' and ' 0.57 ', respectively. These values have now been corrected to ' 0.12 ' and ' 0.73 ', respectively. This has been corrected in both the PDF and HTML versions of the article. The authors would like to apologise for this error.

These authors contributed equally: Xiangyang Zhang, Mi Yang

Xiangyang Zhang

zhangxy@psych.ac.cn

$\triangle$ Huafu Chen

chenhf@uestc.edu.cn

1 CAS Key Laboratory of Mental Health, Institute of Psychology, Chinese Academy of Sciences, Beijing, China

2 Department of Psychology, University of Chinese Academy of Sciences, Beijing, China

3 The Affiliated Brain Hospital of Guangzhou Medical University (Guangzhou Huiai Hospital), Guangzhou, China

4 Department of Stomatology, the Fourth People's Hospital of Chengdu, Chengdu, China

5 Key Laboratory for NeuroInformation of Ministry of Education, School of Life Science and Technology, University of Electronic Science and Technology of China, Chengdu, China

6 Suzhou Psychiatric Hospital, The Affiliated Guangji Hospital of Soochow University, Suzhou, China

7 Psychiatry Research Center, Beijing HuiLongGuan Hospital, Peking University, Beijing, China

8 Department of Psychiatry and Behavioral Sciences, The University of Texas Health Science Center at Houston, Houston, Texas, USA 